\title{
Innovación como campo de estudio en Latinoamérica 2010-2017: Brasil, Chile y México
}

\author{
Rubén Oliver ${ }^{*}$ y Federico Stezano ${ }^{1}$
}

\begin{abstract}
Resumen: Es objetivo de este trabajo explorar a la innovación como tema de estudio en Brasil, Chile y México, durante 2010-2017. Se recurre a la búsqueda de innovation y algunas variantes como tema de búsqueda en la base de datos Scopus. A partir de esa recopilación se ofrecen cuatro indicadores: se identifican áreas de conocimiento que concentran las publicaciones; algún grado de interdisciplinariedad del tema por país, que da una noción de difusión del tema y clústeres de conceptos asociados a la innovación que son relevantes para cada país. Se encuentran diferencias entre países: el tema es más interdisciplinario en México, pero en Brasil se aborda con mayor profusión y sistematicidad con respecto a Chile y México.
\end{abstract}

Palabras clave: innovación; campo de estudio; Latinoamérica.

Abstract: The objective of this work is to explore innovation as a subject of study in Brazil, Chile and Mexico during 2010-2017. It is used to search for innovation and some variants as a search topic in the Scopus database. Based on this collection, four indicators are offered: areas of knowledge are identified that concentrate publications; Some degree of interdisciplinarity of the theme per country, which gives a notion of diffusion of the theme and clusters of concepts associated with innovation that are relevant for each country. There are differences between countries: the subject is more interdisciplinary in Mexico, but in Brazil it is dealt with more profuseness and systematicity with respect to Chile and Mexico.

Keywords: innovation; field of study; Latin America.

Submitted: July $19^{\text {th }} 2017 /$ Approved: November $23^{\text {rd }} 2017$

\section{Introducción}

El estudio de la innovación se remonta a la obra pionera de Schumpeter y, desde entonces, se ha estructurado una comunidad y un ambiente institucionalizado que le da carácter científico y es socialmente organizado. El trabajo de Fagerberg, Fosaas y Sapprasert (2012) explora profusamente la base de conocimiento de la innovación y evidencia la sistematización de su estudio desde los años sesenta del siglo pasado, manifiesto en publicaciones, autores, temas, instituciones académicas y espacios de difusión e identificación de una comunidad temática. Podría decirse que la estructuración de la disciplina ha ocurrido relativamente rápido en años recientes, en virtud de que Fagerberg y Verspagen en 2009 reportaban una organización social temática en ciernes para los estudios de la innovación, en relación con la estructura imperante mejor consolidada en el campo de la gestión estratégica, para los mismos periodos.

La innovación no escapa a la región latinoamericana como objeto de investigación, en razón de lo cual este trabajo tiene por objetivo explorar el concepto como tema de estudio en Brasil, Chile y México, durante el periodo 2010-2017, para tener un acercamiento a los tópicos asociados a la innovación que son relevantes en cada país. ¿Cuál es la relevancia de abordar a la innovación como tema de estudio? ¿Qué se puede decir al respecto a partir de la revisión de las publicaciones científicas, particularmente a partir de los temas asociados a la innovación? ¿Qué puede evidenciarse para el caso de contextos particulares que comparten el mismo objeto de estudio?
Estas interrogantes rondan el presente trabajo y se fundamentan en la necesidad eminentemente académica de identificar el abordaje analítico de la innovación, en función del contexto socio-económico y cultural de su producción.

Esta investigación, basada en información recabada en la base de datos científica Scopus, posee un carácter exploratorio dado que exhibe diferencias significativas entre países por cuanto hace al registro de publicaciones e investigadores. Esto dificulta la posibilidad de ofrecer resultados robustos (como los que podrían provenir de trabajos basados en metodologías de análisis factorial por ejemplo) que permitan alcanzar una mayor profundidad explicativa sobre la incidencia de determinados factores críticos que determinan los ritmos y tendencias de la producción y publicación científica. No obstante, los datos a los que ha podido acceder este trabajo permiten realizar un análisis complejo de la temática con base en cuatro indicadores: (i) uno de conglomeración del tema, en función de las áreas de conocimiento en que se publica el tema innovación; (ii y iiii) dos indicadores que buscan un acercamiento a la difusión del tema, vista desde la interdisciplinariedad y (iv) un último que agrupa clústeres de conceptos asociados a la innovación.

Además de la introducción, en el segundo apartado se habla de la innovación abordada como tema de estudio en dos vertientes: una cognitivo-política y la otra en su vertiente metodológica que deriva de los estudios bibliométricos. La tercera parte expone la metodología de la investigación. Luego se exponen resultados y un apartado final presenta la discusión y conclusiones del trabajo.

Subdirección de Investigación y Posgrado, Centro de Investigaciones Económicas, Administrativas y Sociales, Instituto Politécnico Nacional Ciudad de México, México

*Autor de correspondiencia: neburevilo@gmail.com 


\section{La innovación como tema de estudio: su contexto cognitivo-político y su abordaje metodológico}

\section{Contexto cognitivo-político}

La innovación es un tema de estudio surgido en el ámbito económico y de los negocios, dada su definición como eje de la competencia capitalista en los trabajos seminales de Schumpeter. Su consiguiente configuración como campo disciplinar obedece al interés que suscitó como objeto de investigación y cuya manifestación ha sido núcleo de publicaciones y sus autores ampliamente identificados y validados como constructores de las bases de conocimiento (Fageberg y Verspagen, 2009; Fagerberg, et. al, 2012).

Desde su origen, y con toda claridad actualmente, se puede ubicar a la innovación como tema interdisciplinar, dada su diseminación a diferentes campos de la investigación: la Science Policy Research Unit (SPRU), primera unidad académica orientada hacia el estudio de la innovación, se conformó de un cuerpo académico de variado origen disciplinar: ingenierías, ciencias naturales y ciencias sociales (Fagerber et. al., 2012). Rafols, Leydesdorff, O'Hare, Nightingale y Stirling (2012) ubican la naturaleza interdisciplinar de la innovación y su correlativa inserción en las clasificaciones académicas como tema asociado a temáticas de gestión y negocios. En este trabajo, asimismo, se evidencia que para los tres países latinoamericanos analizados en este estudio la innovación aparece imbricada con áreas temáticas de las ciencias exactas, las naturales, las humanísticas y principalmente, las ciencias sociales.

El proceso por el cual la innovación adquirió su carácter disciplinario se acompañó de un proceso de cimentación de condiciones institucionales respectivas. Tal como reportan Fagerberg, Landström y Martin (2012), luego de que suscitara interés en corporaciones no académicas (como la corporación RAND y la OCDE), el primer esfuerzo por desarrollar la base científica de la innovación ocurrió con la creación de SPRU en 1966, bajo liderazgo de Chris Freeman.

La base institucional de la innovación como tema de estudio es explicable en términos de lo que reportan autores con respecto al surgimiento de otros conceptos que se consolidan como tema de interés científico. Aldrich (2012) para el caso del concepto emprendimiento (entrepreneurship); Clausen, Fagerberg y Gulbrandsen (2012) para el caso de surgimiento de nuevas unidades de investigación (laboratorios, departamentos, entre otras formas) en el marco de campos científicos emergentes; Fagerberg et. Al. (2012) para el caso del concepto sociedad del conocimiento, y Nerur, Rasheed y Natarajan (2008) para el caso de la gestión estratégica, evidencian un patrón de institucionalización de conceptos que radica en una movilización de carácter político-intelectual de los investigadores. Esta movilización se deriva de la identificación del valor cognitivo de un concepto y el consecuente posicionamiento como tema digno de transformarse en objeto de estudio. Ante esta situación, los académicos posicionan el concepto en los ámbitos presupuestario, administrativo, científico, técnico, mediante la organización académica a través de conferencias, seminarios; publicación de libros, revistas; la edificación de laboratorios, departamentos; la institucionalización de consorcios, programas de posgrado, entre otras formas organizativas.

En conjunto, alrededor de un concepto se estructura un complejo organizativo-institucional dinámico, cuya forma y movimiento dependen de cómo los académicos (actores centrales del proceso de creación y desarrollo del campo de conocimiento) conciben el alcance, las vías y la robustez del concepto como objeto de estudio. Y, por tanto, de cómo sus aportes teórico-analíticos y resultados empíricos contribuyen a la producción científica. El establecimiento de un concepto conlleva la estructuración de un discurso y sus medios de difusión, con un trasfondo cognitivo a través del cual ganar adeptos y crear una comunidad. En paralelo, también se detecta una pugna por recursos para producir y difundir conocimiento relacionado a él. Como concepto con una robustez intelectual, se piensa como actividad de largo aliento que, por lo tanto, requiere de formas organizativas manifiestas en escuelas, programas de estudio, departamentos, laboratorios, seminarios.

Por su cuenta, la industria editorial también opera desde su lógica la forma en que la producción científica se organiza para su difusión. En tal medida, contribuye a consolidar conceptos cuyo valor amerita exponerse al mundo científico. Esta situación muestra dos caras de la moneda. La primera es expuesta por Rafols et. al. (2012) quienes señalan que el abordaje de la innovación (dado su carácter interdisciplinario y orientado hacia la solución de problemas desde diferentes unidades de investigación que representan retos institucionales) se distingue por una carencia de paneles de evaluación ajustados a una base disciplinar. De este modo la innovación, comprendida como parte de la investigación en negocios y gestión, suele enfrentar la desventaja de una evaluación sesgada, propia de la investigación interdisciplinaria con respecto a la investigación disciplinaria.

La segunda cara de la moneda es consecuencia de la retroalimentación del mismo complejo organizativo-institucional. Esta forma de difusión del conocimiento obedece a los matices que adquiere el concepto mismo en el marco de las diversas disciplinas para las que observa significatividad. Así, se encuentran publicaciones que específicamente abordan el tema de la innovación a la vez que también se amplía su estudio hacia temas colaterales que se valen de su valor explicativo. Esto potencia el tema en sí mismo, al expandirlo a campos de estudio fuera del ámbito de los negocios y la gestión.

La tensión que subyace en estas distintas perspectivas es reflejo de la lógica social específica del mundo de la ciencia y sus subcampos especializados. En última instancia así se hacen más evidentes los conflictos entre dos fuerzas: (i) las que intentan consolidar en las agendas de los campos científicos los conceptos ya predominantes y (ii) las nuevas nociones, heterodoxas, que cuestionan los conceptos dominantes en el paradigma y construyen nuevas revoluciones científicas en el campo (Merton, 1964). Estas tensiones permanentes, muestran que la elaboración científica implica una elaboración social de la realidad y que el ámbito o campo científico emerge como un espacio conflictivo a su interior y en sus límites con campos cercanos (Bourdieu, 2000). 
En el caso de Latinoamérica, la segunda posguerra generó un ambiente propicio para la institucionalización del estudio de los procesos de desarrollo en ciencia, tecnología e innovación en Argentina Brasil, México y Chile. En esos años distintos apoyos de política crearon facultades de ciencias sociales y consejos nacionales de ciencia y tecnología (Vessuri, 1987: 520-523).

Desde fines de los 60, se consolida un enfoque crítico cuyo discurso enfatiza el análisis del subdesarrollo tecnológico de la región y lleva las temáticas de la relevancia de la ciencia y la tecnología en la sociedad y la industria. Desde mediados de los años 70 y los 80 el interés se comienza a centrar en nuevas temáticas: las instituciones centrales de la innovación, el rol de las comunidades científicas, las evoluciones y tensiones disciplinarias, los análisis de las políticas científicas, así como el estudio de los vínculos existentes entre la innovación tecnológica y la industrialización de la región (ídem: 524-545).

A partir de los finales de los años 80 y los 90 comienzan a re-pensarse los ejes centrales de los estudios latinoamericanos sobre la innovación. En este marco se consolidan los trabajos de corte constructivista, analíticos de procesos de desarrollo científico y tecnológico desde enfoques de redes de conocimiento o de Triple Hélice. En paralelo se impulsa una fuerte producción de estudios de sociología e historia de la ciencia y la tecnología, apoyados en programas de investigación de base empírica. En términos generales además desde esos años hubo un predominio por el estudio de objetos más específicos desde enfoques metodológicos y epistemológicos basados en estudios de caso.

Los temas más recientes de investigación vinculada a la innovación de este siglo muestran similitudes con ciertos temas relevantes para las comunidades europea y norteamericana: la mercantilización de las universidades y el rol de estudios en ciencia y tecnología en las sociedades (Kreimer et al., 2015).

\section{Abordaje metodológico}

Desde una perspectiva metodológica, el estudio del surgimiento y posicionamiento de conceptos como temas de estudio se apoya de la explotación de recursos bibliométricos, cuya base radica en el conteo de ocurrencia y co-ocurrencia de conceptos temáticos y autores, en tres vertientes. La primera vertiente se posiciona en torno a técnicas de análisis factorial, tal como en los trabajos de Di Stefano, Peteraf y Verona (2010) para el estudio del concepto capacidades dinámicas; Nerur, Rasheed y Natarajan (2008) para la identificación de la estructura de la gestión estratégica como campo de estudio y Ponzi (2002) para la identificación de estructura e interdisciplinariedad de la gestión del conocimiento. La segunda vertiente se basa en la representación temática y de autores en mapas a partir de los cuales se identifican temas y autores organizados en clústeres y relaciones entre clústeres, como en los trabajos de Rafols et. al. (2012) para probar la forma en que los rankings de revistas suprimen la investigación interdisciplinaria, y van Eck y Waltman (2010 y 2014), quienes desarrollan un algoritmo para la realización de mapas bibliométricos mediante el programa VOSviewer; en esta misma línea se encuentra el trabajo de Merigó, Cancino, Coronado y Urbyano (2016), quienes a partir del índice $\mathrm{H}$, el conteo de citas, la identificación de nacionalidad de los autores, organizan una clasificación para identificar regiones, países y redes de coautorías por nacionalidad para el tema de la innovación. La tercera vertiente es explotada en el trabajo de Ramos y Ruíz (2004), quienes realizan el conteo de citas para identificar por frecuencia de citas los documentos más relevantes, y el análisis de co-citas para identificar los pares de documentos que son percibidos como similares, mediante el uso del $\mathrm{r}$ de Pearson como medida de similitud, para identificar la estructura intelectual de la investigación en gestión estratégica.

Tanto el análisis factorial, como la definición de medidas de similitud mediante estadísticos como $r$ de Pearson presuponen una mayor robustez en la información recopilada, en términos de la cantidad de publicaciones, citas, co-citas y clusterización de información. Por tanto, tal como se señaló previamente, este trabajo observa un carácter exploratorio, esencialmente con respecto a los conceptos que son relevantes en la investigación sobre innovación. En virtud de lo cual se eligió una estrategia basada primeramente en la conglomeración de áreas de conocimiento que aglutinan el tema de la innovación para dar una noción general del grado de interdisciplinariedad del tema, así como en la agrupación de conceptos en clústeres, para identificar temas que son relevantes en los estudios de la innovación, como a continuación se explicita.

\section{Metodología}

En la base Scopus se buscaron las publicaciones (libros y capítulos; conferencias; artículos de revista) que en el título reportaran las palabras innovation, o innovative, o innovating, o innovate, como criterio conceptual a partir del cual contabilizar el número de publicaciones en temas de innovación, para el periodo de 2010-2017.

Como siguiente criterio de búsqueda se delimitó el país de afiliación del investigador a los países en estudio: Brasil, Chile y México. En este caso, aunque una publicación tenga a varios autores, conque uno de ellos manifieste su filiación en una institución de esos países, sin importar el orden en que aparece como autor, se contabiliza como válida para el conteo de publicaciones que abordan el tema de la innovación.

Con esos criterios de búsqueda, al 8 de abril de 2017 se identificaron 815 publicaciones para Brasil, 87 para Chile y 177 para México (cuadro 1).

Cuadro 1. Tipo de documento

\begin{tabular}{lrrr}
\hline & Brasil & Chile & \multicolumn{2}{c}{ México } \\
Artículo & 597 & 67 & 108 \\
Documento en conferencia & 134 & 14 & 35 \\
Capítulo de libro & & & \\
Libro & 76 & 5 & 27 \\
Total & 8 & 1 & 7 \\
\hline
\end{tabular}

Fuente: Scopus 
A partir de esa búsqueda se elaboran cuatro indicadores, que a continuación se discuten.

\section{Primer indicador: Conglomerados de áreas de conocimiento} donde se concentran las publicaciones

Haciendo uso de las mismas herramientas analíticas de Scopus se identificaron las áreas de conocimiento en que la misma editorial agrupa las publicaciones. Esta agrupación se presenta en los resultados, organizada por conglomerados (tres), organizados mediante el método de vinculación completo, por medición de distancia euclidiana, por lo que la distancia entre clústeres está dada en atención al grado de disparidad entre componentes de los clústeres.

\section{Segundo indicador: factor de interdisciplinariedad}

Luego, a partir del número de publicaciones, a sabiendas de que una misma es clasificada, de acuerdo con el criterio de Scopus, en más de un área de conocimiento, se calculó lo que denominamos factor de interdisciplinariedad, que es el cociente del total de clasificaciones en áreas de conocimiento en que aparecen registradas las publicaciones, entre el total de las publicaciones:

Si planteamos que $a$ es la suma de las publicaciones $x_{i}$

$$
a=\sum x_{i}
$$

Dado que con respecto a $x_{i}$ puede ser posible que

$x_{i} \in A, \mathrm{y} / \mathrm{o} x_{i} \in B, \mathrm{y} / \mathrm{o}$, donde $A$ y $B$ representan áreas temáticas que pueden incluir a una misma publicación $x_{i}$, entonces:

$\sum_{x_{i}} A \cup B \cup \ldots+\sum_{x_{i}} A \cap B \cap$, es igual al número de publicaciones dadas las áreas temáticas A, B... en que se clasifican las publicaciones $X_{i}$, una por una.

De modo que el factor de interdisciplinariedad $c$ es igual a:

$$
C=\frac{\sum x_{i} A \cup B+\sum x_{i} A \cap B}{a}
$$

Ahora bien, dado (1) y que cuando, (2) se transforma:

$$
c=1+\frac{\sum x_{i} A \cap B}{\sum x_{i} A \cup B}
$$

De modo que el factor de interdisciplinariedad ; y a mayor c, mayor interdisciplinariedad, en este caso, de la innovación.

\section{Tercer indicador: Índice de concentración disciplinaria}

Como siguiente indicador, se calcula el índice de concentración disciplinaria $d$, el cual muestra la proporción que representa el área temática $x_{i}$ que con mayor frecuencia registra publicaciones, respecto al número de publicaciones $a$ (ecuación 1), dadas las áreas temáticas en que se clasifican las publicaciones, y que se representa como $y_{i}$

$$
d=\frac{\max _{x_{i} \in a} x_{i}}{y_{i}}
$$

Donde $y_{i}=\sum_{x_{i}} A \cup B \ldots+\sum_{x_{i}} A \cap B \ldots$

\section{Cuarto indicador: clústeres de conceptos}

Para la elaboración de los clústeres de conceptos se recurre al algoritmo del programa VOSviewer. Éste es una herramienta informática para representación gráfica de mapas bibliométricos (citas, co-citas, autores, conceptos), cuya fortaleza y distintivo radica precisamente en que, a diferencia de otros programas, mediante las representaciones robustece la presentación de resultados (van Eck y Waltman, 2010).

Los clústeres de conceptos que se presentan en este trabajo derivan del título de las publicaciones en el tema de la innovación. La organización de conceptos en clústeres se realiza en el programa a través del cálculo de la fuerza de asociación de conceptos, con base en una matriz de co-ocurrencia: dos conceptos co-ocurren si aparecen en un mismo documento y se les otorga un peso relativo con respecto a la ocurrencia total de ambos conceptos en todos los documentos:

$$
s_{i j}=\frac{c_{i j}}{w_{i} w_{j}}
$$

Donde $c_{i j}$ denota número de co-ocurrencias de los conceptos $i$ y $j$, mientras que $w_{i}$ y $w_{j}$ indican el número de ocurrencias de los conceptos $i$ y $j$. La representación de mapas implica el cálculo de otros algoritmos para definición de cercanía de los conceptos, explicados en van Eck y Waltman (2010).

\section{Resultados}

\section{Conglomerados de áreas de conocimiento}

Mediante el programa MiniTab se realizó el ejercicio de conglomeración de áreas de conocimiento en que agrupan las publicaciones de los tres países. Como previamente se señaló, se conglomeró por método de vinculación completa, a través de la medición de distancia euclidiana, lo que gráficamente se muestra en el dendrograma (gráfica 1).

Se decidió organizar la información en tres conglomerados, a partir de las siete áreas de conocimiento en que mayormente se concentran las publicaciones brasileñas, conforme a la información organizada en el cuadro anexo A1. Allí se ve que excepto por lo referente a Ciencia de las Decisiones el resto de las áreas de conocimiento coinciden para los tres países.

Uno de los conglomerados se forma del conjunto de publicaciones que se clasifican en las áreas de Negocios; Gestión y Contabilidad (NGC), y Ciencias sociales (CS), en consecuencia con el origen mismo del concepto en el ámbito de los negocios y sus derivaciones hacia nociones relacionadas con el desarrollo social, la sustentabilidad o la educación, según se verá en los clústeres de conceptos (cuadro 4). Un segundo conglomerado agrupa publicaciones en el área Ciencias de Decisiones (CD), en virtud de que en Brasil es la tercera área de conocimiento que más concentra publicaciones, aunque en Chile esa área de conocimiento es prácticamente marginal (cuadro anexo A1). Ambos conglomerados trazan una perspectiva de la investigación de la innovación en un ámbito empresarial-social. 
El tercer conglomerado, el más nutrido por áreas temáticas, aunque no por cantidad de publicaciones, se forma con las áreas Ingeniería (I); Economía, Econometría y Finanzas (EEF); Ciencias Ambientales (CA), y Ciencias del Cómputo (CC). Es relevante to- mar en consideración de que en este tercer conglomerado el tema de la innovación parece identificarse mayoritariamente con áreas de aplicación técnicas (ingeniería, ciencias ambientales y de cómputo).

Gráfica 1. Conglomerados de las áreas de conocimiento en que se agrupan las publicaciones sobre innovación



Fuente: Elaborado a partir del software MiniTab 17, con datos de publicaciones de la base Scopus

\section{Factor de interdisciplinariedad e índice de concentración disci- plinaria}

De acuerdo con las definiciones que se establecieron de manera previa, el índice de interdisciplinariedad busca establecer una medida de la diversidad de áreas de conocimiento en que se registran las publicaciones para un tema en particular. Se definió que, a mayor índice, un tema tiende a registrarse como interdisciplinario; a menor índice un tema tiende a registrarse como disciplinario. De la comparación entre países, el cálculo del índice señala que en México el tema innovación muestra una mayor interdisciplinariedad (cuadro 2).

En este caso, México es el país cuyas publicaciones en innovación registran una mayor interdisciplinariedad, aunque su índice de concentración disciplinaria es superior al de las publicaciones chilenas.

\begin{tabular}{ccc}
\hline & $\begin{array}{c}\text { Cuadro 2. Factor de interdisciplinariedad e Índice } \\
\text { de concentración disciplinaria }\end{array}$ \\
\hline País & $\begin{array}{c}\text { Factor de } \\
\text { interdisciplinariedad }\end{array}$ & $\begin{array}{c}\text { Índice de concentración } \\
\text { disciplinaria }\end{array}$ \\
Brasil & 1.80 & 0.367 \\
Chile & 1.74 & 0.285 \\
México & 1.84 & 0.291 \\
\hline
\end{tabular}

Fuente: Elaboración propia con base en Scopus

La relevancia de ambos indicadores radica en que pueden manifestar la amplitud de difusión de un tema entre áreas del conocimiento, dado el número de publicaciones. El cuadro A1, por ejemplo, da cuenta de la publicación de trabajos sobre innovación en áreas que aglutinan temas de salud, física y química.

\section{Clústeres de conceptos}

De acuerdo con la información del cuadro 1, la cantidad de publicaciones brasileñas en temas de innovación es mayor 9.4 veces que la chilena y 4.6 veces superior que la mexicana. Consecuentemente, dado que los clústeres se forman a partir de las co-ocurrencias, para un mismo número de éstas, la cantidad de clústeres y sus conceptos asociados para Brasil es superior: a mayor cantidad de publicaciones, la co-ocurrencia de conceptos asociados a la innovación es mayor. De manera que, para efectos de comparación de clústeres y conceptos, se optó por realizar el ejercicio de agrupación de clústeres contando, para el caso de Brasil, a partir de 10 co-ocurrencias; para el caso de Chile de dos y para México de tres. Esto es: a través del programa VOSviewer, se identificaron clústeres a partir de parejas de conceptos que co-ocurren un mínimo de 10 veces para el caso de publicaciones brasileñas, dos veces para el caso de las chilenas y tres para las mexicanas. En resumen, el cuadro 3 muestra la cantidad de conceptos y clústeres identificados a través del programa de cómputo.

Cuadro 3. Conceptos y clústeres: resumen

\begin{tabular}{ccc}
\hline País & Conceptos & Clústeres \\
\hline Brasil & 37 & 7 \\
Chile & 38 & 7 \\
México & 31 & 6 \\
\hline
\end{tabular}

Fuente: Elaborado a partir de VosViewer, con datos de Scopus

El cuadro 4 presenta los clústeres de conceptos derivados de los títulos de las publicaciones que hablan de innovación. Para el caso de Brasil la organización de conceptos en clústeres denota dos intereses 
principales de investigación: (i) en una perspectiva de los negocios y la gestión y las ciencias de decisiones, los conceptos se orientan hacia el estudio de los modelos y estrategias de innovación (clústeres 1, 2 y 3) y (ii) en una perspectiva de las ciencias sociales y la sustentabilidad, se aprecian un conjunto de conceptos asociados a la vertientes sociales de la innovación, como la innovación social, el desarrollo y la sustentabilidad (clústeres 3, 4 y 5).

Para el caso de Chile se destacan dos hallazgos: (i) la agrupación de conceptos en el ámbito de las ciencias sociales y sus vertientes sobre la educación, ciencia, tecnología, así como la innovación y la política pública (clúster 1), (ii) un segundo grupo de conceptos aglutinados en torno a la publicación en áreas de negocios y gestión (clústeres 2, $3,4), 3$ ) referencias sectoriales, como los servicios, en particular los financieros, y las TIC (Clústeres 5 y 6).

Por su cuenta, en el caso de México se manifiesta una producción de publicaciones en una perspectiva de las ciencias sociales manifiesta en la reflexión sobre tema del desarrollo, la ciencia, la tecnología y la política de innovación, con algún grado de imbricación con temas sobre gestión de la innovación (Clústeres 1 y 2) y un grupo de publicaciones cuya base es la gestión de la innovación (Clústeres 3 y 4).

\begin{tabular}{|c|c|c|c|c|c|c|}
\hline \multicolumn{7}{|c|}{ Brasil } \\
\hline Clúster 1 & Clúster 2 & Clúster 3 & Clúster 4 & Clúster 5 & Clúster 6 & Clúster 7 \\
\hline Brazil & Inovao & Innovation & Case & Impact & Knowledge & Science \\
\hline Analysis & Case study & $\begin{array}{l}\text { Knowledge } \\
\text { management }\end{array}$ & Challenge & Practice & Process & Technology \\
\hline Company & Uma & Relationship & Development & $\begin{array}{l}\text { Sustainable } \\
\text { development }\end{array}$ & Research & \\
\hline Brasil & Caso & Study & Management & $\begin{array}{l}\text { Technological } \\
\text { innovation }\end{array}$ & & \\
\hline Innovation system & Estudo & Sustainability & Social innovation & Use & & \\
\hline \multicolumn{7}{|l|}{ Role } \\
\hline \multicolumn{7}{|l|}{ Strategy } \\
\hline \multicolumn{7}{|l|}{$\begin{array}{l}\text { Innovation } \\
\text { management }\end{array}$} \\
\hline \multicolumn{7}{|l|}{ Evidence } \\
\hline \multicolumn{7}{|l|}{ State } \\
\hline \multicolumn{7}{|l|}{ Innovation process } \\
\hline \multicolumn{7}{|l|}{ Contribution } \\
\hline \multicolumn{7}{|c|}{ Chile } \\
\hline Clúster 1 & Clúster 2 & Clúster 3 & Clúster 4 & Clúster 5 & Clúster 6 & Clúster 7 \\
\hline Construction & Approach & Context & Business sustainability & Effect & Chilean model & Case \\
\hline Higher education & Determinant & Efficiency & Challenge & Evidence & Development & Chile \\
\hline Information & $\begin{array}{l}\text { Innovative } \\
\text { practice }\end{array}$ & Innovation & Decade & $\begin{array}{l}\text { Financial } \\
\text { innovation }\end{array}$ & ICT & \\
\hline Innovación & Patent & Knowledge & Lesson & Productivity & TIC & \\
\hline Innovation policy & Practice & Model & Women entrepreneur & Service & & \\
\hline Latin America & Study & Role & & & & \\
\hline Science & $\begin{array}{l}\text { Technological } \\
\text { innovation }\end{array}$ & $\begin{array}{l}\text { Sequential } \\
\text { innovation }\end{array}$ & & & & \\
\hline \multicolumn{7}{|l|}{ Technology } \\
\hline \multicolumn{7}{|c|}{ México } \\
\hline Clúster1 & Clúster 2 & Clúster 3 & Clúster 4 & Clúster 5 & Clúster 6 & \\
\hline Mexico & Technology & Innovación & Performance & Innovation & Design & \\
\hline Development & Science & $\begin{array}{l}\text { Innovation } \\
\text { system }\end{array}$ & Role & Entrepreneurship & Research & \\
\hline case & Innovation policy & Latin America & Knowledge & Sustainability & State & \\
\hline Process & Challenge & $\begin{array}{l}\text { Technology } \\
\text { management }\end{array}$ & Pymes & & & \\
\hline $\begin{array}{l}\text { Technological } \\
\text { innovation }\end{array}$ & $\begin{array}{l}\text { Inclusive } \\
\text { development }\end{array}$ & $\begin{array}{l}\text { Empirical } \\
\text { study }\end{array}$ & & & & \\
\hline Case study & $\begin{array}{l}\text { Latin American } \\
\text { experience }\end{array}$ & $\begin{array}{l}\text { Process } \\
\text { innovation }\end{array}$ & & & & \\
\hline \multicolumn{7}{|l|}{ Lesson } \\
\hline \multicolumn{7}{|l|}{ Eco innovation } \\
\hline Educational innovation & & & & & & \\
\hline
\end{tabular}

Fuente: Elaboración propia, con base en el programa de cómputo VosViewer 


\section{Discusión y conclusiones}

Se argumentó que la conformación de un concepto como tema de investigación deriva de una forma político-cognitiva de proceder de la comunidad científica. Esta, a su vez, es corresponsable de que el tema adquiera peso (por la cantidad de publicaciones que genera) y una condición interdisciplinar (por la diversificación de áreas de conocimiento que reportan publicaciones) como consecuencia del poder explicativo asociado al tema.

Desde esta perspectiva, la conglomeración de las publicaciones en áreas de conocimiento en Brasil, Chile y México, indica una forma de organización del tema por las áreas de conocimiento que implica. Para los tres países, las áreas de conocimiento en que más se aglutinan publicaciones son los negocios y la gestión, así como las ciencias sociales. Asimismo, se evidenció el peso de las publicaciones en el área de las ciencias de decisiones -cuyos vínculos son fuertes con las dos áreas previas- en Brasil. En consecuencia, el denominado índice de concentración disciplinar más elevado corresponde a ese país. Inversamente, el mayor factor de interdisciplinariedad corresponde a México lo que se manifiesta en una configuración de clústeres de conceptos menos concretos (o más difusos) que en Brasil.

Estos datos, en sí mismos, arrojan una aproximación respecto al alcance de la investigación hacia la noción de interdisciplinariedad y difusión del tema: en términos absolutos la cantidad de publicaciones brasileñas es superior al de los otros dos países, pero en términos relativos (en función de la cantidad de publicaciones por país) es un tema tratado menos interdisciplinariamente cuando menos con respecto a México. La misma estructura disciplinaria arroja una identificación más concisa del contenido de los clústeres de conceptos. Si bien éstos se estructuran a partir de un algoritmo, el cálculo no es arbitrario puesto que se forman a partir de enunciaciones significativas (a partir del título de las investigaciones). En el caso de Brasil fue posible identificar tales enunciaciones con mayor claridad.

Dada la forma en que se produce y difunde, el posicionamiento de un concepto como tema de investigación requiere la acumulación de una masa crítica, encarnada en una comunidad de investigación y concretada en condiciones institucionales y organizativas. Estas condiciones son contextuales, de manera que el valor universal de un concepto como tema de estudio enfrente realidades específicas. Estas diferencias son manifiestas en el caso de Brasil, Chile y México con respecto al abordaje de la innovación como tema de estudio: en Brasil la producción científica sobre innovación es más voluminosa que en los otros dos países y allí se observa un mayor índice de concentración disciplinaria y una estructura de conceptos organizados en clústeres más específicos.

En esa perspectiva, los hallazgos de este trabajo ofrecen resultados complementarios al de Kreimer et al (2014) quienes detectan una comunidad científica en torno a un campo científico que crece sistemáticamente, incluyendo a centros de investigación bastante productivos en Argentina, Brasil y México y, en menor medida, a grupos emergentes en años recientes en Colombia, Chile, Venezuela, Uruguay, Perú, Costa Rica y Cuba.
Por su cuenta, la presente investigación observa que para los tres países el tema es relevante en el ámbito de los negocios, pero en Brasil y México es más claro su abordaje en una perspectiva social y de la sustentabilidad. En ambos países, además, se conjugan el interés de investigación tanto en perspectiva macro (sistemas de innovación, por ejemplo) como micro (gestión tecnológica). Otro hallazgo significativo alude a la significación que la producción científica sobre innovación da a la región: para Chile y México Latinoamérica es una referencia, pero no lo es para Brasil.

Los resultados obtenidos en este trabajo ofrecen un diagnóstico sobre la dinámica latinoamericana de producción científica sobre la innovación, con base en la comparación entre países de la región. En términos globales, Latinoamérica observa un relativamente importante crecimiento que la investigación en innovación. No obstante, como señalan Merigó et al (2016) el marcado liderazgo de producción científica de Estados Unidos, seguido de cerca por Reino Unido, pero muy superior a la de los países de Europa occidental y aún más respecto a otras regiones (Europa del Este, Asia, América Latina y África, respectivamente). Los resultados de este trabajo deben ser así interpretados conforme a estas marcadas diferencias inter-regionales de volumen de producción científica sobre innovación, pero especialmente considerando la creciente relevancia que va adquiriendo este tema en América Latina. Dicha importancia se refleja en la cantidad creciente de publicaciones científicas que este trabajo ha identificado en la base de Scopus para Brasil, Chile y México.

\section{Referencias}

Aldrich, H. E. (2012). The emergence of entrepreneurship as an academic field: A personal essay on institutional entrepreneurship. Research Policy, 41(7), 1240-1248. http://dx.doi.org/10.1016/j.respol.2012.03.013.

Bourdieu, P. (2000). Los usos sociales de la ciencia. Buenos Aires: Editorial Nueva Visión.

Clausen, T., Fagerberg, J., \& Gulbrandsen, M. (2012). Mobilizing for change: A study of research units in emerging scientific fields. Research policy, 41(7), 1249-1261. http://dx.doi.org/10.1016/j.respol.2012.03.014.

Di Stefano, G., Peteraf, M. \& Verona, G. (2010). Dynamic capabilities deconstructed: a bibliographic investigation into the origins, development, and future directions of the research domain. Industrial and Corporate Change, 19(4), 1187-1204. http://dx.doi.org/10.1093/icc/ dtq027.

Fagerberg, J., Fosaas, M. \& Sapprasert, K. (2012). Innovation: exploring the knowledge base. Research Policy, 41(7), 1132-1153. http://dx.doi.org/10.1016/j.respol.2012.03.008.

Fagerberg, J., Landström, H. \& Martin, B. (2012). Exploring the emerging knowledge base of "the knowledge society". Research Policy, 41(7), 1121-1131. http://dx.doi.org/10.1016/j.respol.2012.03.007. 
Fagerberg, J. \& Verspagen, B. (2009). Innovation studies-The emerging structure of a new scientific field. Research Policy, 38(2), 218233. http://dx.doi.org/10.1016/j.respol.2008.12.006.

Kreimer, P., Vessuri, H., Velho, L. \& Arellano, A. (2015). Introducción. El Estudio social de la ciencia y la tecnología en América Latina: miradas, logros y desafíos. En P. Kreimer, H. Vessuri, L. Velho y A. Arellano (Eds.), Perspectivas latinoamericanas en el estudio social de la ciencia, la tecnología y la sociedad (pp. 7-27). México: Siglo XXI.

Merigó, J., Cancino, C., Coronado, F. y Urbyano, D. (2016). Academic research in innovation: a country analysis. Scientometrics, 108(2), 559-593. http://dx.doi.org/10.1007/s11192-016-1984-4.

Merton, R. (1964). Teoría y estructura sociales. México: Fondo de Cultura Económica.

Nerur, S., Rasheed, A. \& Natarajan, V. (2008). The intellectual structure of strategic management field: an author and co-citation analysis. Strategic Management Journal, 29(3), 319-336. http://dx.doi.org/10.1002/smj.659.

Ponzi, L. (2002). The intellectual structure and interdisciplinary breadth of Knowledge Management: A bibliometric study of its early stage of development. Scientometrics, 55(2), 259-272. https://link.springer.com/article/10.1023/A:1019619824850.
Rafols, I., Leydesdorff, L., O’Hare, A., Nightingale, P. \& Stirling, A. (2012). How journal rankings can suppress interdisciplinary research: A comparison between Innovation Studies and Business \& Management. Research Policy 41(7), 1262-1282. http://dx.doi.org/10.1016/j. respol.2012.03.015.

Ramos-Rodríguez, A. \& Ruíz-Navarro, J. (2004). Changes in the intellectual structure of strategic management research: a bibliometric study of the Strategic Management Journal, 1980-2000. Strategic Management Journal, 25(10), 981-1004. http://dx.doi.org/10.1002/ smj.397.

Van Eck, N.J. \& Waltman, L. (2010). Software survey: VOSviewer, a computer program for bibliometric mapping. Scientometrics, 84(2), 523-538. http://dx.doi.org/10.1007/s11192-009-0146-3.

Van Eck, N.J. \& Waltman, L. (2014). Visualizing bibliometric networks. In Y. Ding, R. Rousseau, \& D. Wolfram (Eds.), Measuring scholarly impact: Methods and practice (pp. 285-320). Switzerland: Springer.

Vessuri, H. (1987). The Social Study of Science in Latin America. Social Studies of Science, 17(3), 519-554. 


\section{Cuadro anexo A1}

Áreas de conocimiento donde aparecen publicaciones sobre innovación (Cantidad de publicaciones por país)

\begin{tabular}{|c|c|c|c|c|c|}
\hline Brasil & & Chile & & México & \\
\hline Business, Management and Accounting & 538 & Business, Management and Accounting & 43 & $\begin{array}{l}\text { Business, Management and } \\
\text { Accounting }\end{array}$ & 95 \\
\hline Social Sciences & 227 & Social Sciences & 32 & Social Sciences & 70 \\
\hline Decision Sciences & 189 & Economics, Econometrics and Finance & 29 & $\begin{array}{l}\text { Economics, Econometrics and Fi- } \\
\text { nance }\end{array}$ & 43 \\
\hline Engineering & 117 & Engineering & 12 & Environmental Science & 26 \\
\hline Economics, Econometrics and Finance & 113 & Computer Science & 6 & Engineering & 19 \\
\hline Environmental Science & 90 & Environmental Science & 6 & Computer Science & 18 \\
\hline Computer Science & 50 & Psychology & 4 & Decision Sciences & 17 \\
\hline Energy & 27 & Agricultural and Biological Sciences & 3 & Arts and Humanities & 8 \\
\hline Medicine & 24 & Arts and Humanities & 3 & Agricultural and Biological Sciences & 6 \\
\hline Agricultural and Biological Sciences & 22 & Decision Sciences & 3 & Energy & 6 \\
\hline Arts and Humanities & 22 & Energy & 3 & Medicine & 6 \\
\hline Mathematics & 9 & Mathematics & 3 & Chemical Engineering & 5 \\
\hline Earth and Planetary Sciences & 8 & Chemical Engineering & 1 & Psychology & 3 \\
\hline Psychology & 8 & Earth and Planetary Sciences & 1 & Chemistry & 2 \\
\hline Chemical Engineering & 6 & Immunology and Microbiology & 1 & Health Professions & 1 \\
\hline Biochemistry, Genetics and Molecular Biology & 3 & Medicine & 1 & Immunology and Microbiology & 1 \\
\hline Chemistry & 3 & & & & \\
\hline Materials Science & 3 & & & & \\
\hline Physics and Astronomy & 3 & & & & \\
\hline Pharmacology, Toxicology and Pharmaceutics & 2 & & & & \\
\hline Health Professions & 1 & & & & \\
\hline Immunology and Microbiology & 1 & & & & \\
\hline Nursing & 1 & & & & \\
\hline
\end{tabular}

Fuente: Elaborado a partir de Scopus 
